\title{
A regional massive hemorrhage protocol developed through a modified Delphi technique
}

Jeannie L. Callum MD, Calvin H. Yeh MD PhD, Andrew Petrosoniak MD, Mark J. McVey MD, Stephanie Cope, Troy Thompson BAHSc, Victoria Chin BSc, Keyvan Karkouti MD, Avery B. Nathens MD, Kimmo Murto MD, Suzanne Beno MD, Jacob Pendergrast MD, Andrew McDonald MD, Russell MacDonald MD, Neill K.J. Adhikari MD, Asim Alam MD, Donald Arnold MD, Lee Barratt NP, Andrew Beckett MD, Sue Brenneman RN, Hina Razzaq Chaudhry MLT, Allison Collins MD, Margaret Harvey, Jacinthe Lampron MD, Clarita Margarido MD, Amanda McFarlan RN, Barto Nascimento MD, Wendy Owens BComm, Menaka Pai MD, Sandro Rizoli MD, Theodora Ruijs MD, Robert Skeate MD, Teresa Skelton MD, Michelle Sholzberg MD, Kelly Syer RN, Jami-Lynn Viveiros MLT, Josee Theriault MD, Alan Tinmouth MD, Rardi Van Heest MD, Susan White MLT, Michelle Zeller MD, Katerina Pavenski MD

\section{Abstract}

Background: A massive hemorrhage protocol (MHP) enables rapid delivery of blood components in a patient who is exsanguinating pending definitive hemorrhage control, but there is variability in MHP implementation rates, content and compliance owing to challenges presented by infrequent activation, variable team performance and patient acuity. The goal of this project was to identify the key evidence-based principles and quality indicators required to develop a standardized regional MHP.

Methods: A modified Delphi consensus technique was performed in the spring and summer of 2018. Panellists used survey links to independently review and rate (on a 7-point Likert scale) 43 statements and 8 quality indicators drafted by a steering committee composed of transfusion medicine specialists and technologists, and trauma physicians. External stakeholder input from all hospitals in Ontario was sought.

Results: Three rounds were held with 36 experts from diverse clinical backgrounds. Consensus was reached for 42 statements and 8 quality indicators. Additional modifications from external stakeholders were incorporated to form the foundation for the proposed MHP.

Interpretation: This MHP template will provide the basis for the design of an MHP toolkit, including specific recommendations for pediatric and obstetrical patients, and for hospitals with limited availability of blood components or means to achieve definitive hemorrhage control. We believe that harmonization of MHPs in our region will simplify training, increase uptake of evidence-based interventions, enhance communication, improve patient comfort and safety, and, ultimately, improve patient outcomes.

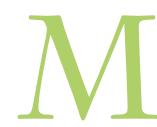
assive bleeding is a leading preventable cause of death following trauma, childbirth and surgery. ${ }^{1-3}$ There were 5.1 million deaths after traumatic injury worldwide in 2010 , mostly affecting young people, accounting for nearly $10 \%$ of all deaths. ${ }^{4}$ In the United States, it is estimated that up to $20 \%$ of such deaths are the direct result of preventable hemorrhage. ${ }^{4-7}$ Management of unstable hemorrhagic shock is centred on stabilizing the patient with prompt transfusion of blood components, and rapid identification and treatment of the source of bleeding. Patient outcome is dependent on the availability of rapid definitive surgical intervention, support of a transfusion medicine and clinical laboratory, prompt access to hemostatic agents and care provided by a high-performing interdisciplinary team. ${ }^{8}$ In the trauma literature, protocolized delivery of massive transfusion streamlines the complexities

Competing interests: For panellists and conflicts of interest, see Appendix 1 (available at www.cmajopen.ca/content/7/3/E546/suppl/DC1).

This article has been peer reviewed.

Correspondence to: Jeannie Callum, Jeannie.Callum@sunnybrook.ca CMAJ Open 2019. DOI:10.9778/cmajo.20190042 
of rapid access to surgical care and blood components, decreases variability of treatment, increases protocol compliance, reduces blood component wastage, facilitates interprofessional communication and allows for tracking of metrics for continuous quality improvement. ${ }^{9-12}$

Most academic institutions have a massive hemorrhage protocol (MHP) in place to rapidly deliver blood components and coordinate care in the setting of traumatic injury. ${ }^{13}$ However, in a recent survey of 150 hospitals in Ontario, the proportion of hospitals with MHPs in nonacademic settings was lower than that in academic settings, and there was significant protocol heterogeneity between hospitals. ${ }^{14}$ This was also seen in well-established trauma centres in the US,,$^{15}$ where civilian implementation is still highly variable. ${ }^{12}$ Compliance with MHPs is also highly variable, ${ }^{12}$ and low compliance may have ramifications for patient outcomes. ${ }^{16}$ A systematic review of before-after studies comparing patients with trauma managed in periods with and without an MHP showed an association between MHP use and better survival. ${ }^{17}$ The benefits of an MHP have not been tested in rigorous prospective randomized trials. Maintaining high levels of compliance with MHPs appears to be a universal challenge. ${ }^{12,16}$ Ontario has the largest population of all the Canadian provinces and provides hospital care in a diverse mixture of settings with high and low health care resources. Massive hemorrhage is an infrequent event in many areas served by small regional hospitals. As a result, access to blood components, laboratory tests of hemostasis and surgical expertise are highly variable. Instituting an adaptable MHP for the province based on the local health care resource setting is needed to streamline the complex logistics of rapid delivery of blood components, facilitate rapid patient transfer where required and reduce the cognitive burden on bedside clinicians. With the ultimate goal of developing such a protocol, we aimed to identify the key principles and quality indicators required to develop a provincial standardized evidence-based MHP template for hospitals.

\section{Methods}

\section{Steering committee and panellists}

A steering committee composed of transfusion medicine specialists and technologists, and trauma physicians selected the panel members, organized the MHP forum, administered the surveys and analyzed the results. The steering committee assembled a panel of 36 content experts to represent relevant stakeholders. The panel members were selected through the Ontario Regional Blood Coordinating Network, which oversees transfusion use, audits of practice and educational initiatives for the region. The steering committee selected members for their broad expertise and responsibility in administering MHPs across Ontario's geographically diverse network. Panel participation was voluntary and not financially remunerated. Travel reimbursement and meals were provided, and authorship was afforded only to those who participated in all phases of the Delphi process. The panel included anesthesiologists, trauma sur- geons, obstetricians, hematologists, transfusion medicine physicians, emergency physicians, prehospital and transport medicine physicians, intensivists, blood supplier representatives, nurses, technologists and a patient. The group represented the geographically diverse health care programs in the province, with representatives from academic hospitals, specialized pediatric institutions, suburban hospitals and smaller, rural hospitals.

The panellists were informed of the purpose and scope of the exercise, and of the requirement to attend a 2-day MHP forum in Toronto and complete all rounds of the Delphi exercise. To standardize the knowledge base of panellists, they were provided with copies of original papers selected by the steering committee and reflecting the most up-to-date evidence in the area of massive hemorrhage management before the first round. The MHP forum had both didactic and interactive sessions, in which content experts reviewed each area of the MHP for 15 minutes, after which there was a 15-minute discussion period for the attendees.

\section{Study design}

We chose a modified Delphi technique to establish consensus and the framework for the provincial MHP toolkit. The Delphi technique is a systematic, interactive method that relies on a panel of experts to converge on consensus statements following a series of iterative surveys. ${ }^{18}$ Rounds of surveys are continued until consensus is achieved. The Delphi technique is deemed a relevant source of evidence in health care research and is particularly important if randomized controlled trials are unavailable to set health care policies. ${ }^{19}$ Our group used this method previously ${ }^{20}$ and noted its ability to costeffectively include a large number of participants who are geographically dispersed, while preventing unequal representation or biasing of opinions in the consensus through iterations of anonymous voting. ${ }^{21}$ We modified the Delphi technique to allow for open forum of discussion in round 1 only, to ensure that the broadest range of expert experience was captured in the consensus.

The modified Delphi rounds were conducted independently by each panellist via an emailed survey link (LimeSurvey). Survey responses were anonymized before centralized review. Each statement was independently rated on a 7-point Likert scale from "Definitely should not include" to "Definitely should include" in the MHP, with cut-offs based on previously established Delphi criteria. ${ }^{20}$ There was an "opt out" option for each statement to account for possible lack of expertise in a specific area ("Unable to rate as outside area of expertise"). Panellists were asked to provide suggestions to enhance statement clarity with each numerical ranking. Panellists were instructed to answer questions on the basis of what they considered optimal patient care or best practices, rather than what they believed was currently operationally feasible at their institution.

The first round consisted of 43 statements and 8 quality indicators that had been drafted by the steering committee based on available literature and existing MHPs. The 
statements were based on the core tenets of existing MHPs, such as rapid and reliable access to blood components, tertiary care transfer and use of antifibrinolytic agents, and the quality indicators were based on quality measures established by the National Surgical Quality Improvement Program. The first round was conducted 1 month before the MHP forum. The survey responses of the first round were anonymized and presented to the panellists and the steering committee on day 2 of the MHP forum for discussion of the phrasing and structure of statements scoring below a median Likert score of 5.5 (see a priori criteria below), as the modification to the Delphi methodology. Round 1 provided panellists with the ability to add statements and quality indicators. To ensure nonbiased and independent statement review, panellists were not provided the ratings and survey comments of the other panel members. After round 1, no further inperson meetings or formal interactions between panellists took place.

\section{Criteria for disposition of items}

A priori criteria for disposition of the items in the first round were established as follows:

1. Items receiving a median Likert score of at least 5.5 (out of 7) would be accepted as written and not subject to further rounds. These statements were to be incorporated into a provincial MHP as written, unless a clear improvement in phrasing was suggested by a panellist that would not change the intent of the item.

2. Items with a median score of 2.6 to 5.4 were to be discussed at the in-person meeting with all the panellists. Following discussion, the items would be revised by the steering committee and sent out electronically in the second round.

3. Items with a median score of 2.5 or less were to be removed from further rounds, unless there was strong opposition by the panel, in which case a revision would be drafted for the second round.

4. Panellists were provided with a comment box to allow for addition of novel statements and quality indicators on the first round and were allowed to suggest additional statements and quality indicators at the in-person meeting. No additional statements were added after round 2 .

A priori criteria for disposition of the items in the second or later round were established as follows:

1. Items receiving a median Likert score of at least 5.5 would be accepted as written and not subject to further rounds, unless a clear improvement in phrasing was suggested by a panellist that would not change the intent of the item.

2. Items with a median score of 2.5 to 5.4 were to be rewritten on the basis of comments by the panellists and sent out in the third or subsequent round.

3. Items with a median score of 2.4 or less were to be removed from further rounds of scoring.

4. Where suggested by panellists and/or the steering committee, merging or division of statements could occur where appropriate.
After consensus was reached in the final Delphi round, the statements were circulated via email to the medical directors of transfusion medicine responsible for 150 of the 262 Ontario hospitals with licensed transfusion laboratories. The accompanying letter included a request to distribute the statements to members of their hospital transfusion committees and MHP leaders for feedback. These 304 people represented the clinical and technical leads of the laboratories. All hospitals in Ontario able to issue red blood cells have a medical director who is registered with the provincial Ministry of Health and Long-Term Care, which provided us with an upto-date email contact list. Feedback was collated and recommendations were incorporated by the steering committee where necessary to improve the clarity of the statements and their justification.

The initial drafting of the consensus statements was completed by March 2018. Round 1 of the consensus panel was completed on Apr. 13, 2018, and was discussed in person on Apr. 21, 2018 in the MHP forum. Rounds 2 and 3 were subsequently completed in June 2018 and August 2018, respectively.

\section{Ethics approval}

Ethics approval was not required for this Delphi exercise.

\section{Results}

Of the 44 experts invited to participate, 36 agreed (Appendix 1, available at www.cmajopen.ca/content/7/3/E546/suppl/DC1). A $100 \%$ response rate was achieved from panellists in all 3 rounds of the modified Delphi exercise. The progression toward consensus based on Likert scores is shown in Figure 1, and the overall degree of consensus for each statement by the last round is shown in Figure 2. Consensus was achieved for all statements by round 3, yielding a final consensus document with 42 statements and 8 quality indicators. Owing to lack of consensus on round 1 , statements $7,22,23$ and 34 were broken into their individual components for rounds 2 and 3 to assist with understanding the components driving the lack of consensus (Figure 1). Unless otherwise specified, all statements and quality metrics also apply to a pediatric MHP. The statements together with a brief rationale for each are presented in Table 1, in a logical order rather than in order of clinical importance.

Four areas required additional rounds and major modifications: 1) selection of the name of the protocol, 2) selection of the laboratory resuscitation targets, 3 ) determination of the pack configurations and 4) clarification of the role of recombinant factor VIIa. The primary obstacle to selecting a name for the protocol was that many hospitals already had long-standing MHPs with specific names. Consensus on the laboratory targets and pack configuration was achieved in the third round by splitting statements into subsections. The recombinant factor VIIa statement required 3 rounds of review to ensure that the phrasing mitigated the panellists' apprehensions regarding this controversial therapy. Following the generation of consensus statements, no 


\begin{tabular}{|c|c|c|c|}
\hline Statement & $\begin{array}{c}\text { Round 1: median score } \\
\text { (range; abstaining votes) }\end{array}$ & $\begin{array}{c}\text { Round 2: median score (range; } \\
\text { abstaining votes) or counts } \\
\text { where appropriate }\end{array}$ & $\begin{array}{l}\text { Round 3: median score (range; abstaining } \\
\text { votes) or counts where appropriate }\end{array}$ \\
\hline 1 & $7(5-7 ; 1)$ & & \\
\hline 2 & $7(5-7 ; 1)$ & & \\
\hline 3 & $7(4-7 ; 3)$ & & \\
\hline 4 & $7(1-7 ; 1)$ & & \\
\hline 5 & $6(2-7 ; 2)$ & $7(4-7 ; 3)$ & \\
\hline 6 & $7(5-7 ; 1)$ & & \\
\hline 7 & $6(2-7 ; 2)$ & $6(1-7 ; 1)$ & Overhead "Code Transfusion": rank 1 \\
\hline 8 & $6(3-7 ; 1)$ & & \\
\hline 9 & $7(4-7 ; 2)$ & & \\
\hline \multirow[t]{2}{*}{10} & $6(2-7 ; 4)$ & \multirow[b]{2}{*}{$7(5-7 ; 1)$} & \\
\hline & $7(4-7 ; 4)$ & & \\
\hline 11 & $7(4-7 ; 1)$ & & \\
\hline 12 & $7(3-7 ; 1)$ & & \\
\hline 13 & $7(3-7 ; 1)$ & & \\
\hline 14 & $6(2-7 ; 4)$ & $7(2-7 ; 1)$ & \\
\hline 15 & $7(3-7 ; 2)$ & & \\
\hline 16 & $7(3-7 ; 1)$ & & \\
\hline 17 & $7(1-7 ; 1)$ & $7(2-7 ; 1)$ & \\
\hline 18 & $7(4-7 ; 2)$ & & \\
\hline 19 & NA & $7(1-7 ; 0)$ & \\
\hline 20 & $7(4-7 ; 2)$ & & \\
\hline 21 & $6(2-7 ; 1)$ & & \\
\hline 22 & $7(2-7 ; 1)$ & $\begin{array}{l}\text { "Yes" to need for test: } \\
\text { - CBC, INR, iCalcium 36/36; } \\
\text { fibrinogen 35/36; pH 34/36 } \\
\text { - aPTT 29/36; electrolytes 32/36; } \\
\text { lactate 30/36 }\end{array}$ & \\
\hline \multirow[t]{2}{*}{23} & \multirow[t]{2}{*}{$7(2-7 ; 3)$} & $\begin{array}{l}\text { - Hemoglobin > } 80 \mathrm{~g} / \mathrm{L} 31 / 36 \\
\text { - } \text { INR }<1.826 / 36 \\
\text { - Fibrinogen }>1.5 \mathrm{~g} / \mathrm{L} 24 / 36 \\
\text { - Platelets }>50 \times 10^{9} / \mathrm{L} 26 / 36 \\
\text { - iCalcium > } 1.15 \mathrm{mmol} / \mathrm{L} 17 / 36\end{array}$ & \\
\hline & & $\begin{array}{l}\text { - INR }<1.511 / 36 \\
\text { - Fibrinogen }>2.0 \mathrm{~g} / \mathrm{L} 12 / 36 \\
\text { - Platelets }>100 \times 10 \% / \mathrm{L} 12 / 36 \\
\text { - iCalcium }>1 \mathrm{mmol} / \mathrm{L} 11 / 36\end{array}$ & \\
\hline 24 & $7(4-7 ; 5)$ & $7(5-7 ; 4)$ & \\
\hline 25 & $7(4-7 ; 4)$ & & \\
\hline 26 & $7(4-7 ; 7)$ & & \\
\hline 27 & $7(2-7 ; 4)$ & & \\
\hline 28 & $6(4-7 ; 3)$ & $7(2-7 ; 1)$ & \\
\hline 29 & $7(2-7 ; 5)$ & & \\
\hline 30 & $7(4-7 ; 1)$ & & \\
\hline 31 & $7(2-7 ; 1)$ & $7(6-7 ; 1)$ & \\
\hline 32 & $7(2-7 ; 11)$ & $7(5-7 ; 5)$ & \\
\hline 33 & $7(1-7 ; 3)$ & & \\
\hline \multirow[t]{7}{*}{34} & \multirow{7}{*}{$6(3-7 ; 4)$} & \multirow[t]{7}{*}{$6(1-7 ; 4)$} & Platelet transfusion based on count: $7(1-7 ; 4)$ \\
\hline & & & Communicate if no platelet transfusion: $6(2-7 ; 4)$ \\
\hline & & & Box $1: 7(1-7 ; 2)$ \\
\hline & & & Box 2: $7(2-7 ; 4)$ \\
\hline & & & Box $3: 7(1-7 ; 4)$ \\
\hline & & & Small hospital box 2: $7(2-7 ; 5)$ \\
\hline & & & Convert to laboratory-guided: $7(2-7 ; 5)$ \\
\hline 35 & $7(3-7 ; 8)$ & $7(2-7 ; 6)$ & \\
\hline 36 & $7(4-7 ; 6)$ & $7(5-7 ; 6)$ & \\
\hline 37 & $6(1-7 ; 11)$ & $7(3-7 ; 4)$ & \\
\hline 38 & $7(1-7 ; 1)$ & & \\
\hline 39 & $7(1-7 ; 2)$ & $7(4-7 ; 1)$ & \\
\hline \multirow[t]{2}{*}{40} & $6(4-7 ; 5)$ & \multirow[b]{2}{*}{$7(4-7 ; 4)$} & \\
\hline & $\begin{array}{c}6(2-7 ; 8) \\
6.5(2-7 ; 5)\end{array}$ & & \\
\hline 41 & $7(5-7 ; 1)$ & & \\
\hline $42-1$ & $7(1-7 ; 4)$ & $7(4-7 ; 2)$ & \\
\hline $42-2$ & $7(1-7 ; 2)$ & $7(2-7 ; 1)$ & \\
\hline $42-3$ & $6(1-7 ; 5)$ & $7(5-7 ; 1)$ & \\
\hline $42-4$ & $7(1-7 ; 6)$ & $7(4-7 ; 1)$ & \\
\hline $42-5$ & $6(1-7 ; 2)$ & $7(1-7 ; 1)$ & \\
\hline $42-6$ & $6.5(1-7 ; 2)$ & $7(4-7 ; 1)$ & \\
\hline $43-7$ & $7(1-7 ; 2)$ & $7(4-7 ; 1)$ & \\
\hline $42-8$ & $6(1-7 ; 2)$ & $7(3-7 ; 1)$ & \\
\hline
\end{tabular}

Figure 1: Median scores on a 7-point Likert scale for the 3 rounds of the Delphi exercise. Counts are provided for cases in which panellists were asked to rank or choose between options rather than use the Likert scale. Green = passed with minor or no phrasing adjustment (empty green box = passed on a preceding round); red = statements that did not pass based on numerical scoring and/or critical written comments, resulting in a major content revision; gray = statements that were not accepted and were then merged for the subsequent round; blue = a new statement that was added after round 1. Split rows denote the need for division of the statement into its components for the scoring round. Note: CBC $=$ complete blood count, iCalcium = ionized calcium, INR = international normalized ratio, NA = not applicable, aPTT = activated partial thromboplastin time. 


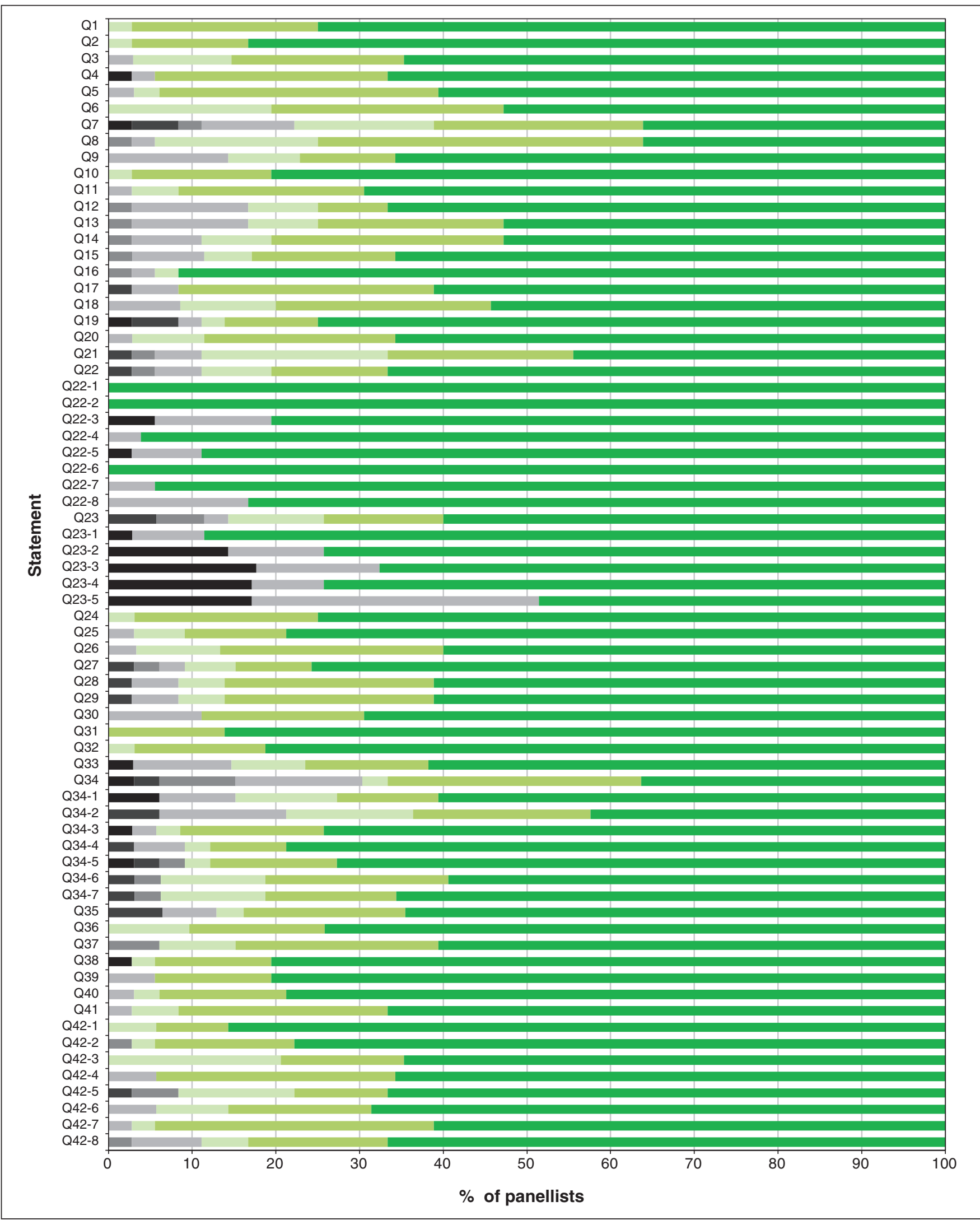

Figure 2: Proportion of Likert scores at the time of the final consensus round for each statement. Panellists were asked to indicate agreement on a 7-point Likert scale $(7=$ highest level of agreement). Black and gray $=$ scores less than 5 ; green shades $=$ scores of 5 or more. Responses of "no," "uncertain" and "yes" are denoted as black, gray and green, respectively. 
substantive changes to the statements were made, but modifications were required to clarify the supporting text.

The external review by the medical directors of transfusion did not necessitate changes to the statements; however, the feedback provided valuable input to strengthen the supporting text and provide input on logistical and implementation challenges. All statements and the contents of the manuscript were approved by all authors and panellists.

Table 1 (part 1 of 7 ): Massive hemorrhage protocol statements and rationale

Statement

1. All hospitals shall have a protocol to guide the management of a massively bleeding patient.

2. The protocol shall be developed by a multidisciplinary team and approved by the hospital transfusion

3. The protocol shall incorporate the principles of damage-control resuscitation, specifically giving highest priority to treating the source of hemorrhage.

4. The protocol shall consider the available resources at the institution. committee (or other relevant multidisciplinary committee).

Rationale

The panel concluded that an MHP is required to standardize the approach to the massively bleeding patient for all hospitals. For the purposes of the MHP, a hospital is defined as any organization that either maintains a red blood cell inventory or staffs an emergency department, urgent care centre, critical care unit, labour and delivery unit, or operating room. The panel recognized there are small clinic facilities where a bleeding patient may be encountered but where transfusion is currently not available and an MHP would not be appropriate. The panel concluded that a policy for rapid transport of patients with massive hemorrhage to a facility with an MHP would be required at such a facility.

The MHP requires support from multiple hospital services including, but not limited to, emergency, trauma, surgery, anesthesiology, critical care, blood transport personnel, communication services and laboratory personnel. ${ }^{11}$ The protocol should be reviewed and approved by the hospital transfusion committee (or other relevant hospital committee) and the medical advisory committee.

Damage-control resuscitation principles in traumatic injury include abbreviated surgical and/or endovascular interventions for hemorrhage control and management of intra-abdominal contamination, and critical care support to correct deranged physiologic measures (hypothermia, acidosis, coagulopathy), with definitive surgical repair delayed until stabilization and hemostatic control have been achieved. ${ }^{22}$ In the severely injured trauma population, damage-control resuscitation is associated with reduced mortality, although the approach has never been tested in a randomized controlled trial. ${ }^{17,23,24}$ Ongoing hemorrhage leads to worsening coagulopathy and other physiologic derangements. ${ }^{25}$ Although the role of damage-control resuscitation outside of traumatic injury is unknown, prompt hemorrhage control is likely to be an important component of care..$^{26,27}$

The hospital must consider the available resources of the institution when developing the local protocol. Centres caring for pediatric patients should ensure personnel are prepared for weight-based dosing and the use of size-specific equipment (e.g., warming devices, intravenous infusion equipment). Smaller and more remote hospitals located at a distance from the blood supplier will need to make adjustments to streamline their MHP to compensate for the limited number of team members, blood component inventory and laboratory testing menus, and the ability to provide definitive surgical or endovascular control of hemorrhage. The MHP will need to specify, if required, which patients should be transferred in a timely manner to other facilities for definitive treatment and how this should be achieved. Examples for simplification for smaller/remote sites include 1) prelabelled uncrossmatched RBC units ready for immediate transfusion, 2) preprepared laboratory sample collection kits, 3) administration of a single bolus of tranexamic acid rather than an infusion, 4) administration of PCCs and fibrinogen concentrate instead of plasma and cryoprecipitate, 5) use of point-of-care technology for laboratory testing and 6) cross-training hospital personnel from other patient care areas.

5. A single protocol for all patients is preferred in order to ensure compliance; there should be specific guidance provided for selected patient populations (e.g., obstetrical patients should receive early fibrinogen replacement).

A survey from academic hospitals showed that $60 \%$ of respondents have a single protocol for all patients. ${ }^{28}$ Compliance with a single MHP is poor in published studies, ${ }^{12,16,29}$ which raises the concern that consistent care would be further compromised by multiple protocols for different bleeding scenarios. The panel recommended a single, standardized protocol in response to the massively bleeding patient with options to tailor the protocol for specific patient populations. For example, in massive obstetrical hemorrhage, consideration should be given to measuring fibrinogen levels early and repeatedly, administering fibrinogen replacement if the level falls below $2.0 \mathrm{~g} / \mathrm{L}^{30}$ and using an intrauterine balloon device as a bridge to definitive bleeding control. ${ }^{31}$ In gastrointestinal hemorrhage, consideration should be given to prompt endoscopic therapy for hemorrhage control. ${ }^{32,33}$ In hemorrhage after cardiac surgery, there is evidence to support the use of viscoelastic testing (as compared to standard laboratory tests) in reducing the risk of major bleeding. ${ }^{34}$ Pediatric patients require weightbased dosing of blood components and hemostatic adjuncts, consideration of potentially higher transfusion triggers depending on comorbidities and age, and provider awareness of increased risk or hyperkalemia and hypothermia. ${ }^{35-38}$

6. The protocol should be reviewed The science and clinical trial activity in the area of massive hemorrhage, coagulopathy and MHPs are at a minimum of every 3 years.
The science and clinical trial activity in the area of massive hemorrhage, coagulopathy and MHPs are
rapidly evolving. Each institutional MHP should be reviewed at a minimum of every 3 years to ensure alignment with the scientific evidence and the provincial MHP. The protocol revision should be conducted by a multidisciplinary team as detailed in statement 2 , and approved by the hospital transfusion committee and medical advisory committee. 
Table 1 (part 2 of 7): Massive hemorrhage protocol statements and rationale

Statement

Rationale

7. The protocol shall be called "The Massive Hemorrhage Protocol" and, if activated as an overhead announcement, referred to as "Code Transfusion."

8. Participating team members should have access to formal training and drills to increase awareness, adherence and effective delivery of the MHP.

9. The written MHP should be readily accessible as a reference tool for all team members.

10. The transport service(s) should be promptly notified if the decision is made to transfer the patient to another hospital for definitive hemorrhage control. If required, the patient should be transferred as soon as and as safely as possible by appropriate staff and transport resources, to an institution where definitive hemorrhage control can be performed.

11. The protocol shall have activation criteria.
The existence of several different terms for the protocol across Ontario has created confusion and delays to activation (e.g., a trainee calling communications to activate the Code Omega protocol at a hospital that activates the protocol by calling the transfusion medicine laboratory to activate the "massive transfusion protocol"). The panel, after much deliberation, chose the protocol name "Massive Hemorrhage Protocol" for the following reasons: 1) massive transfusion is most commonly defined in adults as a transfusion of 10 or more units of RBCs in a 24-hour period; however, some patients will not survive to receive 10 units, and many patients who receive 4-10 units need additional therapies contained in an MHP; 2) the name highlights the importance of definitive hemorrhage control; and 3) an MHP is more than just a transfusion protocol and includes nontransfusion interventions (e.g., maintenance of normothermia, use of antifibrinolytics). The panel agreed that the method for MHP activation should be site-specific and be clearly defined in the protocol but that if a hospital-wide overhead announcement was implemented, a standard term should be used at all institutions. The consensus term chosen by the panel is "Code Transfusion" owing to its clarity, ease of pronunciation and lack of overlap phonetically with other "colour" codes (e.g., Code Bleed or Code Blood with Code Blue). The value of an overhead announcement is that it provides redundancy if the paging system fails and notifies all hospital employees that the laboratory is under acute pressure (and to refrain from calling for nonemergency blood products and nonurgent test results).

To achieve high levels of team performance and protocol adherence, team members require access to formal training material and exposure to multidisciplinary drills or simulations. This is particularly important for high-stress and rarely encountered massive hemorrhage scenarios. Simulations have been successfully employed for training in obstetrical hemorrhage, ${ }^{39}$ pediatric hemorrhage ${ }^{40}$ and trauma ${ }^{41} \mathrm{~A}$ systematic review of 33 studies involving 1203 resident and medical student participants showed that simulation was associated with improved provider behaviour and patient outcomes. ${ }^{42}$ In a systematic review of 13 studies of trauma team training, both nontechnical skills and team-based performance improved. ${ }^{43}$ Importantly, these improvements extend to patient outcomes, as simulationbased training is associated with improved outcomes in trauma and cardiac arrest care. ${ }^{44,45}$

To achieve high levels of protocol compliance among staff, ready access to the MHP is required. The local institution should develop resources (either in electronic or paper format) to assist clinicians with MHP compliance. The format and medium should be dictated by the local hospital circumstances.

There are 150 hospitals in Ontario that have access to transfusion support. Owing to Ontario's large geographic size and numerous remote regions, it would not be possible to have large stocks of blood components available at all hospitals without very high levels of wastage. Timely evacuation of massively bleeding patients from smaller centres to larger centres capable of definitive hemorrhage control is needed for 2 reasons: 1) the small blood stocks held in remote hospitals (typically a small number of RBCs, no platelet pools and limited stocks of frozen plasma) and 2) lack of access to definitive surgical or radiologic intervention to allow for hemorrhage control. There is little published on evacuation time targets within civilian settings. Rapid evacuation (<60 min) among military patients with trauma who had noncompressible torso injury and amputation injury was associated with reduced mortality ${ }^{46}$ Clinicians working with limited capacity to achieve surgical hemostasis should aim to transfer as soon and as safely as possible.

Underactivation (i.e., delayed or no activation of MHP for patients who require hemorrhage control and blood components) could be catastrophic, as it may result in otherwise preventable exsanguination. A retrospective study suggested that delay in initial blood component administration is associated with worse outcomes (each 1-min delay to the arrival of the first pack of blood components is associated with a $5 \%$ increase in the risk of death). ${ }^{47}$ In contrast, overactivation (i.e., MHP activation that is ultimately not required) may lead to unnecessary transfusion, wastage of blood components and diversion of human resources away from competing needs. Despite concern that appropriate and timely activation are critical, there are no criteria with both high sensitivity and high specificity for predicting the need for massive transfusion. The 2 most commonly used scores validated in this setting are the Shock Index (blood pressure divided by heart rate or modified pediatric Shock Index ${ }^{48}$ ) and the ABC score (1 point each for penetrating injury, blood pressure $\leq 90 \mathrm{~mm} \mathrm{Hg}$, heart rate $\geq 120$ beats/min and positive results of FAST [Focused Assessment with Sonography for Trauma]), with the Shock Index performing slightly better than the $A B C$ score in traumatic injury. ${ }^{49} \mathrm{New}$ data suggest that resuscitation intensity ( $\geq 4$ units of fluid in the first $30 \mathrm{~min}$, with 1 unit defined as any of 1 unit of RBCs, 1 unit of plasma, $500 \mathrm{~mL}$ of colloid or $1 \mathrm{~L}$ of crystalloid) may represent an important alternative metric to identify patients who require MHP activation..$^{50}$ In pediatric patients, a retrospective study of combat-injured children defined massive transfusion as the requirement for $40 \mathrm{~mL} / \mathrm{kg}$ or more of blood components transfused within 24 hours. ${ }^{51}$ Given the current lack of evidence to support one set of activation criteria over another, the activation criteria should be set by the hospital to meet the needs of the local patient population. 
12. The protocol shall have termination criteria.

13. The protocol shall specify the team members required to respond when the protocol is activated.

14. The protocol should specify how the lead clinician at the bedside is designated.

15. The protocol shall specify the team member(s) designated to be responsible for blood component and sample transport.

16. The transfusion medicine laboratory and the core laboratory shall be notified of all MHP activations.

17. All critical laboratory results and important coagulation parameters (hemoglobin, platelet count, INR and fibrinogen) shall be communicated verbally to the clinical team as soon as they are available.

18. The timing of protocol activation and termination shall be recorded in the patient's chart.
Termination of the protocol allows personnel to return unused blood components to regular inventory, cease ordering blood components from the blood supplier, cease thawing of frozen components and divert resources to competing needs. In contrast, premature termination may lead to a reduction in the number of team members at the bedside, in the frequency of laboratory testing and in the availability of blood components. Termination should be considered when bleeding source control has been attained, hemodynamic stability has been achieved, vasopressor requirements have diminished, and the transfusion rate has slowed such that additional transport personnel are no longer required. Typically, when these features are present, transfusion decisions can be guided by laboratory test results. ${ }^{52}$ As no explicit criteria have been validated, termination criteria should be determined at the local hospital level. The method to communicate the termination of the MHP should be specified in the local hospital protocol.

Executing all the necessary tasks specified in an MHP, in addition to all the other clinical tasks required to achieve surgical control of blood loss, will require mobilization of an interdisciplinary team. The precise composition of the clinical team can be modified according to the acuity of the hemorrhage, the location of the patient, the type of hemorrhage and the institution's available resources. For example, the neonatal team will be required to attend postpartum hemorrhages to provide immediate care for the neonate, whereas in trauma MHPs managed in the trauma room, where nursing to patient ratios are already high, additional nursing staff may not be required. Given the association between survival and the time of arrival of the first cooler of blood components, a dedicated transport team for both blood samples and components is critical.

How the lead clinician for the MHP is assigned should be specified in the local hospital protocol as it will be highly variable depending on the patient population served and the institutional resources. A broad range of physicians could serve as the team leader. In addition, in smaller organizations without on-site physicians, a nurse practitioner or midwife may be the most appropriate team leader. There may be a transition in leadership as the patient moves from one location to another. The process of handover from one leader to the next should be explicitly stated in the protocol. There must be training in nontechnical skills for the team leads to promote high performance for communication, situational awareness and decision-making skills. In simulation training, higher performance on nontechnical skills by the team lead (situational awareness and decision-making) correlates with critical task completion and improved team performance..$^{53}$ Simulation training for clinicians leading trauma resuscitation improves confidence and reduces anxiety. ${ }^{54}$ The provision of formal feedback to trauma team leaders in training by faculty is associated with improvement in leadership skills over time. ${ }^{55}$

The protocol shall specify the team members designated to be responsible for both the transportation of blood components and patient blood samples for laboratory testing. Although the protocol specifies the use of ratio-based resuscitation (standardized RBCs to plasma) to mitigate the risk of coagulopathy, this does not prevent overtransfusion or provide assurance that coagulation competence will be maintained. ${ }^{56}$ Early and repeated laboratory testing (with rapid transportation of the samples to the laboratory) to confirm adequacy of transfusion resuscitation is required. It is also critical that blood components be rapidly supplied to the bedside and that empty coolers be returned to the transfusion medicine laboratory.

Early and prompt notification of the transfusion medicine laboratory will assist with timely blood component delivery, rapid transition to group-specific blood and designation of the transfusion medicine technologist team leader. A single individual on the clinical side should be the sole source of contact between the clinical team and the transfusion medicine technologist leader so as to reduce the risk of duplicate transfusion orders. Activation of the core laboratory technologists will ensure designation of the laboratory technologist team leader, rapid identification of MHP samples, prioritization of the testing, complete testing of all required tests for the MHP and immediate communication of test results to the clinical team.

During MHP activation, the clinical team may not have ready access to the electronic health record owing to patient acuity and clinical area layout. It is therefore required that all critical results (preliminary or complete, and as defined by the local laboratory) and important coagulation results (hemoglobin, platelet count, INR and fibrinogen) be verbally communicated to the clinical team as soon as the results are available. This may mitigate the risks of undertransfusion or overtransfusion, and improve time to correction of other biochemical derangements (hyperkalemia, hypocalcemia, acidosis). The "push of information" is thought to be an important tool to improve team performance. ${ }^{57}$ Consideration should be given to having dedicated mobile phones to mitigate the risk of communication failure between the laboratory and the clinical team due to rapid movement of the clinical team from one hospital location to another.

Documentation of the activation and termination times must be recorded in the patient chart in the format specified by the local institutional policy. This could be documented by hand or electronically in the nursing or physician notes, or in the electronic computerized physician ordering system. These times are necessary during the review of the patient chart for the purposes of quality improvement. 


\section{Table 1 (part 4 of 7): Massive hemorrhage protocol statements and rationale}

Statement

19. Patients and/or their substitute decision-maker for whom the massive hemorrhage protocol was activated should be informed. Actual (e.g., transfusionassociated circulatory overload, hyperkalemia) and potential adverse effects should be disclosed. Furthermore, women of child-bearing potential should be informed of the risk of red blood cell alloimmunization.

20. The collection and testing of the group and screen sample shall be prioritized in the protocol to mitigate the impact on group $\mathrm{O}$ red blood cells and $A B$ plasma stocks.

\section{Laboratory testing should be} done at baseline and at a minimum hourly until the protocol is terminated.

22. The recommended minimum laboratory testing (where the test is available) at each blood draw should be: CBC, INR, activated partial thromboplastin time (aPTT; baseline only), fibrinogen, electrolytes, calcium (ionized), blood gas ( $\mathrm{pH}$ and base excess) and lactate.

\section{The protocol should state the} minimum laboratory protocol resuscitation targets for transfusion: 1) hemoglobin > $80 \mathrm{~g} / \mathrm{L}$ (RBC); 2) INR $<1.8$ (plasma or prothrombin complex concentrates); 3) fibrinogen $>1.5 \mathrm{~g} / \mathrm{L}$ (cryoprecipitate or fibrinogen concentrates); 4) platelets $>50 \times$ $10^{9} / \mathrm{L}$; 5) ionized calcium $>1.15 \mathrm{mmol} / \mathrm{L}$. Relevant transfusion targets can also be used if viscoelastic testing is performed.

24. All massively bleeding patients should have a temperature measured within 15 minutes of arrival or protocol activation, and then at a minimum of every 30 minutes (or continuously where available) until the protocol is terminated.

\author{
Rationale
}

At the earliest possible opportunity, the most responsible physician (or delegate) must have a conversation with the patient and/or the substitute decision-maker regarding why the MHP was activated, the number and types of components transfused, the transfusion complications observed and the potential long-term consequences of transfusion. Informed consent for transfusion should be obtained as per local hospital policy. Patients have variable perceptions related to transfusion risks, ${ }^{58}$ and accurate communication of the potential risks is important to achieve patient-centred care. Women of child-bearing potential should be informed of the risk of red blood cell alloimmunization, which may result in hemolytic disease of the fetus and newborn, and should be counselled to undergo red blood cell antibody screening 6 weeks and/or 6 months after transfusion (many antibodies are evanescent, and there is a brief window for detection)..$^{59}$

Both group O RBCs and AB plasma are in chronic short supply in Canada. The proportion of group $\mathrm{O}$ RBCs transfused to non-group-O recipients is increasing, with trauma accounting for $10 \%$ of this pressure on group $O$ blood stocks. ${ }^{60}$ The vast majority of $A B$ plasma units are transfused to non- $A B$ recipients. ${ }^{61}$ Given the pressure on $A B$ plasma stocks, it has not been possible to provide male-only $A B$ plasma for all recipients, with resultant cases of transfusion-related acute lung injury from female $A B$ plasma. ${ }^{62}$ Hence, the draw of the group and screen sample, rapid transport of the sample to the laboratory and testing of the sample should be prioritized.

See rationale for statement 22.

Baseline laboratory testing is prognostic, ${ }^{63}$ identifies patients receiving oral anticoagulant therapy in need of reversal and directs immediate need for components in excess of the base ratio of RBCs to plasma. Although the proposed MHP includes the use of early ratio-based resuscitation for plasma before laboratory test results of coagulation are available, this does not guarantee that coagulopathy will be prevented and raises the risk of transfusion of unnecessary blood components. Laboratory confirmation of adequate hemostatic resuscitation is required at least hourly. Current guidelines recommend early and repeated measures of hematology and coagulation parameters. ${ }^{24}$ The measurement of aPTT is recommended only at baseline to detect the anticoagulant effect of certain anticoagulants (e.g., dabigatran) and preexisting bleeding disorders (e.g., hemophilia). If the baseline INR and aPTT are correlated, further aPTT measures are not indicated and may in fact delay release of the other coagulation test results. ${ }^{64}$ Increased aPTT in postpartum hemorrhage is associated with worse outcomes; however, there is considerable overlap and minimal difference between outcome groups, so elevated aPTT is not clinically useful. ${ }^{30}$ Transitioning from blind ratio-based component therapy to one based on conventional laboratory testing or point-of-care viscoelastic testing has the potential to minimize unnecessary transfusions and allow for targeted component therapy. ${ }^{34}$

Biochemical tests (e.g., potassium, calcium and $\mathrm{pH}$ ) may indicate potential complications from massive transfusion or inadequate resuscitation of the patient in hemorrhagic shock. Lactate measurements are also predictive of mortality, although the role of serial measurements in improving patient outcomes has not been confirmed in clinical trials. ${ }^{65}$

As there are no prospective studies evaluating laboratory resuscitation targets in the setting of massive bleeding, the suggested laboratory targets are based on the consensus opinion of the panellists and are concordant with the published literature. ${ }^{24,66}$ These are minimum targets to be maintained throughout the resuscitation and are not meant to be overly prescriptive (i.e., restricting blood component issue based on the above values). Certain pediatric populations, such as neonates, those with congenital heart disease, those receiving extracorporeal life support and those in severe respiratory distress, may require higher thresholds for RBC transfusion during an MHP. ${ }^{36-38}$ 
Table 1 (part 5 of 7 ): Massive hemorrhage protocol statements and rationale

Statement

Rationale

25. All patients should receive interventions to prevent

hypothermia and achieve

normothermia $\left(\geq 36^{\circ} \mathrm{C}\right)$.

26. All patients should receive warmed intravenous fluids, red blood cells and plasma to avoid hypothermia.

27. Red blood cells should be delivered in a validated container to prevent wastage.

28. The MHP protocol should ensure there are processes in place to ensure an uninterrupted supply of blood components to the bedside.

29. If the blood group is unknown, O Rh D-negative red blood cells should be used only for female patients of child-bearing potential (age $<45 \mathrm{yr}$ )

30. Uncrossmatched red blood cells shall be available at the bedside within 10 minutes of MHP activation.

31. In bleeding patients in need of red blood cell transfusion, uncrossmatched red blood cells should be transfused until crossmatch-compatible red blood cells are available.

32. There is no threshold of units of group $\mathrm{O}$ red blood cells above which a switch to group-specific red blood cells is prohibited. The switch to group-specific red blood cells and plasma should be done as soon as possible.

33. The protocol shall state the reversal strategy for commonly used orally administered

anticoagulants.
See rationale for statement 26 .

In both traumatic injury and postpartum hemorrhage, temperature monitoring is infrequently performed, and, when the temperature is measured, hypothermia is common. ${ }^{67,68}$ Hypothermia in traumatic injury is associated with worse outcomes, ${ }^{69,70}$ although prospective trials have not confirmed whether aggressive warming protocols would alter outcomes. ${ }^{71}$ Mild hypothermia is associated with a $22 \%$ increase in the risk of transfusion. ${ }^{72}$ Warming of patients improves their comfort, and, therefore, even in the absence of a confirmed survival benefit, it should be a core part of every MHP. ${ }^{73}$

Since RBCs are a valuable resource, strategies to reduce wastage during transport to and storage at the patient bedside are required. Numerous investigators have validated that wastage can be mitigated with appropriate temperature-controlled devices, with resultant substantial cost savings. ${ }^{74,75}$ At large academic centres with frequent MHP activation, all components should be transported in validated containers to mitigate component wastage.

The local MHP should include processes to ensure an uninterrupted supply of blood components to the bedside until termination. Specifically, the next cooler should be brought to the patient location before the previous cooler is empty. This will minimize the risk of lacking necessary blood components during the resuscitation. The person assigned to maintain the uninterrupted supply of blood components should be specified in the protocol. The procedure for requesting the next set of blood components should be stated in the protocol, easy to perform in the setting of massive hemorrhage and designed with the intention of preventing wrong-patient transfusion errors. The delivery of blood components to the bedside should not be equated with an order for transfusion.

O Rh D-negative stocks are insufficient to allow all patients of unknown blood group to be supported with O Rh D-negative RBCs until the blood group result is released in the laboratory information system. The risk of alloimmunization in an Rh D-negative patient after exposure to Rh D-positive RBCs in the setting of major bleeding is $20 \% .^{76,77}$ Immunization to the D-antigen is relevant only for females who wish to have future pregnancies. Over $99 \%$ of births occur among women less than age 45 years ${ }^{78}$ and hospital MHPs should restrict the use of O Rh D-negative RBCs for women less than this age. For conscious women, efforts should be made to determine their age early in the course of care so that the transfusion medicine service can be instructed to supply the optimal Rh D-type of blood. The risk of immunization from Rh D-positive platelets is $1 \%$, and therefore Rh-immunoglobulin should be provided only to Rh D-negative women less than age 45 (after transfer to the intensive care unit but within $72 \mathrm{~h}$ of the Rh D-incompatible platelet transfusion). ${ }^{79}$

See rationale for statement 31 .

In retrospective analyses in trauma resuscitation, faster time to delivery of the first pack of RBCs was associated with superior survival (every 1-min delay to the first pack was associated with a $5 \%$ increase in the odds of mortality). ${ }^{47}$ Collection of the group and screen sample, transport of the sample to the laboratory, centrifugation of the sample, testing and result release into the laboratory information system require about 70-90 minutes. Therefore, following MHP activation, it is not appropriate to wait for crossmatch-compatible RBCs. The transfusion laboratory must have a protocol and process for the immediate release of uncrossmatched RBCs. In severe traumatic injury, in cases in which communication from the prehospital emergency services suggests the patient will need immediate transfusion owing to hemodynamic instability and severe injury, it is appropriate to order RBCs to the emergency department in advance of patient arrival.

Each unit of RBCs in Canada is produced with a minimal amount of residual plasma ( $<30 \mathrm{~mL}$ per unit), and, therefore, even after transfusion of 10-20 units of group O RBCs, the amount of incompatible plasma is trivial and does not preclude a transition to group-specific RBCs.

The MHP protocol shall include a table with all approved anticoagulant therapies and their appropriate reversal strategy, including the dosage(s) of the therapies to be administered. 


\section{Table 1 (part 6 of 7): Massive hemorrhage protocol statements and rationale}

Statement

34. The initial management of the rapidly bleeding patient that precludes the use of laboratoryguided transfusion should begin with immediate red blood cell (RBC) transfusion and then transfusions at an RBC:plasma ratio of 2:1.

\section{Rationale}

Two prospective randomized trials failed to confirm a survival benefit of an RBC:plasma ratio of 1:1 (compared to 2:1). ${ }^{80,81} \mathrm{~A}$ large retrospective review of experience before and after implementation of 1:1 resuscitation failed to show a mortality benefit. ${ }^{22}$ The Canadian consensus conference on massive transfusion recommended an RBC:plasma ratio of 2:1 followed by transition to laboratory-guided administration of blood components as soon as possible. ${ }^{83}$ The standard approach outlined below, which is based on expert consensus, is applicable to most large adult hospitals. No blood components should be transfused without a clear order and specified infusion rate from the team leader or delegate. Simplified options are provided for institutions that do not stock plasma, platelets and/or cryoprecipitate (or are unable to provide the components in a rapid manner owing to limited numbers of personnel or lack of thawing devices), or that cannot provide definitive surgical or radiological care, and the goal is to stabilize in preparation for transport by land or air ambulance. Pediatric institutions should develop age- and weight-based MHP component protocols to ensure that blood components and fractionated coagulation factors are delivered in appropriate ratios. In institutions that care for pediatric patients, the transfusion boxes must come with clear instructions for the clinical team to mitigate the risk of over- or undertransfusion.

Standard approach

1. Box 1 should contain 4 RBC.

2. Box 2 should contain 4 RBC, 4 plasma.

3. Box $3^{*}$ should contain 4 RBC, 2 plasma and fibrinogen replacement (10 units of cryoprecipitate or $4 \mathrm{~g}$ of fibrinogen concentrate).

4. Platelets, when stocked in the hospital transfusion laboratory, should be transfused based on the platelet count.

Simplified options for smaller organizations

1. As per standard approach.

2. Box 2 (where plasma not stocked in hospital transfusion laboratory) should contain 4 RBC, 2000 IU of PCC and $4 \mathrm{~g}$ of fibrinogen concentrate. Efforts should be made to transfer the bleeding patient to a centre capable of definitive hemorrhage control.

3. As per standard approach.

4. Platelets, when not stocked in the hospital transfusion laboratory, should be ordered in for transfusion (if patient cannot be promptly transferred out). If the patient is transferred before platelets are transfused, this should be communicated to the receiving hospital.

35. Recombinant factor VIIa (rVIIa) should be considered only when massive hemorrhage is refractory to surgical hemostasis, medical optimization of coagulation parameters, acidosis and hypocalcemia, and be used in consultation with an expert in the management of coagulopathy in the massively bleeding patient.

36. Fibrinogen concentrate, $4 \mathrm{~g}$ (equivalent to approximately 10 units of cryoprecipitate), can be used as a reasonable alternative to cryoprecipitate for fibrinogen replacement.
Recombinant activated factor VIIa ( $\mathrm{VVIIa}$ ) has not been shown to reduce mortality in prospective randomized controlled trials. ${ }^{84,85}$ In contrast, rVIla is associated with an increase in thromboembolic complications. ${ }^{84}$ Given the concerns regarding lack of efficacy and potential risks, all other lower-risk hemostatic therapies should be exhausted, and rVIla should be used only in consultation with an expert in the management of coagulopathy of the massively bleeding patient.
37. At institutions lacking sufficient resources to issue plasma (e.g., no thawing device or no plasma stocked in inventory), prothrombin complex concentrates (PCCs), 2000 IU, can be substituted for coagulation factor replacement. Fibrinogen replacement should be given concurrently with PCCs unless the fibrinogen level is known to be $\geq 1.5 \mathrm{~g} / \mathrm{L}$.
Cryoprecipitate in Canada is provided as individual units that must be thawed, reconstituted with saline and then pooled. This takes about 30-45 minutes of technologists' time and may compete with their ability to perform laboratory testing or prepare other components. The product can be kept for only 1 year after donation. It must be transported frozen at all times. Once thawed and pooled, it expires after 4 hours. Given the time-intensive preparation requirements and limited shelf life, it is reasonable for some hospitals to transition to pathogen-reduced fibrinogen concentrates. There are no large randomized controlled trials of cryoprecipitate and fibrinogen concentrates to determine equivalence, although a large trial in cardiac-surgery-related hemorrhage (FIBrinogen REplenishment in Surgery [FIBRES] trial) is ongoing. ${ }^{86}$ For pediatric patients, a dosage of about $50 \mathrm{mg} / \mathrm{kg}$ of fibrinogen concentrate up to a maximum of $4 \mathrm{~g}$ is suggested. ${ }^{87}$

Similar to the challenges with cryoprecipitate, some smaller organizations may have challenges in providing plasma during an MHP (no thawing device or not stocked in the laboratory owing to rarity of use). In these situations, a reasonable option is to transfuse PCCs and fibrinogen concentrates. This is a common strategy used in many European countries, and outcomes appear to be similar to those with a plasma resuscitation strategy in trauma, usually guided by viscoelastic point-of-care testing. ${ }^{88}$ This strategy should be seen as a bridge before transport to an institution capable of definitive surgical management and more complete transfusion support. For pediatric patients, a dosage of $25 \mathrm{IU} / \mathrm{kg}$ of PCCs (rounded to the closest $500 \mathrm{IU}$ ) up to a maximum of $2000 \mathrm{IU}$ is suggested. ${ }^{89,90}$ 
Table 1 (part 7 of 7): Massive hemorrhage protocol statements and rationale

Statement

Rationale

38. Pretransfusion bedside patient and product identification check shall be performed before transfusion of any component to avoid mistransfusion.

39. Patient demographics shall not be updated/changed until after termination of the protocol. Once the MHP is terminated, patient demographics should be updated as soon as possible.

40. Tranexamic acid should be administered as soon as intravenous or intraosseous access is achieved but within 3 hours from time of injury or within 3 hours from MHP activation in all other patients.
Transfusion-related errors remain common in the emergency department. ${ }^{91,92}$ Under no circumstances can the pretransfusion bedside patient and product identification check be aborted, especially in mass-casualty scenarios, where there may be multiple patients receiving blood components simultaneously.

Patients admitted during major hemorrhage or after traumatic injury are frequently registered with a temporary name and number (e.g., "Unidentified," "Andrew") or with an incomplete registration (e.g., no date of birth). Modifications to key identifiers during active resuscitation may delay the issue of blood components from the transfusion service or result in an erroneous incompatibility detected at the pretransfusion bedside check. The update of the patient identification should be delayed until the patient's condition has stabilized and with coordination between the nursing team and the transfusion medicine laboratory to ensure no gaps in release of laboratory test results or transfusion support.

Tranexamic acid reduces mortality in the setting of trauma ${ }^{93}$ and postpartum hemorrhage ${ }^{94}$ It is most effective when given immediately, with the survival benefit decreasing by $10 \%$ for every 15 -minute delay in administration and with no benefit after 3 hours from injury/onset of bleeding. ${ }^{95}$ There is no increased risk of venous or arterial thromboembolic complications ${ }^{96}$ Dosages and infusion rates vary depending on the study protocol (1-g bolus plus 1-g infusion over $8 \mathrm{~h},{ }^{93} 1 \mathrm{~g}$ bolus and $1 \mathrm{~g}$ bolus repeated at $1 \mathrm{~h},{ }^{97}$ 1 -g bolus and repeated if ongoing bleeding at $\geq 30 \mathrm{~min}^{94}$ and $2-\mathrm{g}$ bolus at the scene of the injury (Clinicaltrials.gov; NCT01990768; trial completed). The dosage and infusion rate should be determined by the local institution. Simplification may be needed in more resource challenged locations, and a single 2-g bolus may be preferred. Evidence of the efficacy of tranexamic acid in pediatric trauma is currently limited, but its use in pediatric patients with trauma requiring transfusion is accepted practice, within the same time parameters as for adults. For pediatric patients, the initial bolus of tranexamic acid can be dosed at $15 \mathrm{mg} / \mathrm{kg}$ up to a maximum of $1 \mathrm{~g}$, with additional doses/infusion based on local policy. ${ }^{98,99}$ The role of tranexamic acid in gastrointestinal bleeding has not been confirmed; a large multicentre trial (Haemorrhage Alleviation With Tranexamic Acid - Intestinal System [HALT-IT]) is under way to determine whether tranexamic acid assists with hemostasis and reduces transfusion or mortality rates. ${ }^{100}$ Tranexamic acid should be readily available in clinical areas where massive hemorrhage is common to prevent delays in administration.

41. MHP activations should be reviewed by a multidisciplinary committee for quality assurance.
Compliance with MHPs is poor during the resuscitation of a critically ill patient who has multiple competing priorities. ${ }^{12,16}$ Implementation of an MHP is just the first step to improving the care of massively bleeding patients; training, simulations, checklists, audit and feedback are needed to achieve high levels of performance. At a minimum, the quality metrics listed in Box 1 should be tracked on consecutive MHP activations by a multidisciplinary team, with feedback to the front-line staff at regular intervals.

\footnotetext{
42. The quality metrics in Box 1 should be tracked on all activations of the protocol and the data reviewed quarterly at the hospital transfusion committee and the medical advisory committee.
}

Note: aPTT = activated partial thromboplastin time, INR = international normalized ratio, MHP = massive hemorrhage protocol, PCC = prothrombin complex concentrate, $\mathrm{RBC}=$ red blood cell.

${ }^{*}$ Few patients will require more than 12 units of RBCs for an acute hemorrhage. In patients who have received 12 or more units of RBCs, transfusion decisions for plasma and fibrinogen replacement should be made based on hourly measurement of the INR and fibrinogen levels, and orders communicated promptly to the blood bank.

\section{Interpretation}

Through a modified Delphi iterative process, we selected and refined 42 statements and 8 quality indicators to form the foundation for the proposed provincial standardized, evidence-based MHP template for hospitals. The MHP toolkit will include training material, simulation exercises, checklists, template policies and procedures, and patient material. In addition to decreasing variability in care, reducing cognitive load on providers, improving communication between the clinical and laboratory teams, increasing uptake of evidence-based treatments and ultimately improving patient outcomes, we hope that this initiative will improve patient comfort and safety, communication with families and disclosure of transfusion risks to patients. Although the lists of statements are not exhaustive, we hope that they address the current widespread variability in MHP structure. ${ }^{14,15}$ Both the modified Delphi process and the external stakeholder consultation assisted with statement construction to ensure clarity for both experts in transfusion medicine and health care personnel working outside of the laboratory.

This consensus document created by the modified Delphi method is a powerful tool that is broadly applicable and adaptable to many hospital settings. The consensus panel included excellent representation by resource-limited and geographically constrained sites in rural Ontario, as well as resource-rich 


\begin{tabular}{|c|c|c|}
\hline \multicolumn{3}{|c|}{ Box 1: Quality metrics to be tracked on all activations of the protocol } \\
\hline Quality metric & $\begin{array}{l}\text { Local } \\
\text { reporting }\end{array}$ & $\begin{array}{l}\text { Provincial } \\
\text { reporting }\end{array}$ \\
\hline $\begin{array}{l}\text { Q1. Proportion of patients receiving tranexamic acid within } 1 \mathrm{~h} \\
\text { of protocol activation }\end{array}$ & $\mathrm{X}$ & $\mathrm{X}$ \\
\hline $\begin{array}{l}\text { Q2. Proportion of patients in whom RBC transfusion is initiated } \\
\text { within } 15 \text { min of protocol activation }\end{array}$ & $\mathrm{x}$ & $\mathrm{X}$ \\
\hline $\begin{array}{l}\text { Q3. Proportion of patients (of patients requiring transfer for } \\
\text { definitive care) with initiation of call for transfer within } 60 \mathrm{~min} \\
\text { of protocol activation }\end{array}$ & $\mathrm{X}$ & \\
\hline $\begin{array}{l}\text { Q4. Proportion of patients achieving temperature } \geq 35^{\circ} \mathrm{C} \text { at } \\
\text { termination of the protocol }\end{array}$ & $\mathrm{X}$ & \\
\hline $\begin{array}{l}\text { Q5. Proportion of patients with hemoglobin levels maintained } \\
\text { between } 60 \text { and } 110 \mathrm{~g} / \mathrm{L} \text { during protocol activation, excluding } \\
\text { certain pediatric populations (e.g., neonates) that may require } \\
\text { higher hemoglobin values }\end{array}$ & $\mathrm{X}$ & \\
\hline $\begin{array}{l}\text { Q6. Proportion of patients transitioned to group-specific red blood } \\
\text { cells and plasma within } 90 \text { min of arrival/onset of hemorrhage }\end{array}$ & $\mathrm{x}$ & $\mathrm{x}$ \\
\hline $\begin{array}{l}\text { Q7. Proportion of patients with appropriate activation ( } \geq 6 \mathrm{RBC} \\
\text { units in first } 24 \mathrm{~h},>40 \mathrm{~mL} / \mathrm{kg} \text { per } 24 \mathrm{~h} \text { of RBCs in pediatric } \\
\text { patients) or before this level in patients dying due to hemorrhage } \\
\text { within } 24 \mathrm{~h}\end{array}$ & $\mathrm{X}$ & \\
\hline $\begin{array}{l}\text { Q8. Proportion of patients without any blood component } \\
\text { wastage (including plasma that is thawed and not used within } \\
\text { the 5-day limit on another patient) }\end{array}$ & $\mathrm{x}$ & \\
\hline
\end{tabular}

tertiary care centres. As a result, the recommendations reflect best practice but are also flexible, as they include modifiers for clinical environments with fewer team members and limited access to laboratory testing and blood product availability. For example, the MHP recommends that centres without the ability for definitive hemorrhage control call for early transport (statements 1 and 10), facilities where certain blood components are limited are offered alternatives (statement 34), and there is dosage modification for the initial antifibrinolytic agent for patients in remote locations to ensure administration of a timely and complete dose (statement 40). In comparison, a recent survey of hospitals with MHPs in the United States showed that the majority were in large academic centres, calling for a higher ratio of plasma:red blood cells (1:1), with very few unified MHPs in place to encompass nontrauma indications. ${ }^{101}$

The Delphi technique was chosen as the method to develop consensus statements from expert stakeholders because of the absence of clinical trial evidence for all aspects of the management of the massively bleeding patient. ${ }^{102}$ Although clinical trials provide considerable guidance on the utility of blood component ratios, ${ }^{80}$ antifibrinolytic agents, ${ }^{93,94}$ use of recombinant factor $\mathrm{VII}^{84,85}$ and other areas of management, they fail to provide recommendations on how to construct the protocol, modifications for community hospitals or specific patient populations., ${ }^{912,26}$ We modified the Delphi exercise to allow for an open forum after round 1 to increase input from experienced practitioners and to discuss the vast quantity of available literature to ensure that all participants had a foundation in MHP knowledge. This allowed for the broadest capture of areas of massive hemorrhage that are logistical in nature and do not lend themselves to evaluation in clinical trials, such as communication strategies and procedures, protocol nomenclature, frequency and type of laboratory testing, laboratory resuscitation targets, and blood component transport and bedside storage. ${ }^{26}$ Importantly, modification of the Delphi exercise also allowed the invaluable input of a patient representative, who provided insight into the importance of patient-related outcomes such as communication and hypothermia management to ensure comfort.

\section{Limitations}

The major limitations and challenges of our modified Delphi process included the limited number of panellists per specialty and hospital type (e.g., obstetrics, remote hospitals) owing to cost and logistical limitations. Furthermore, 1 panellist ranked the statements based on feasibility and/or cost of recommendations despite instructions to rank based on best practices; however, later in round 1 , the panellist reranked based on best practices. Another limitation was the failure to include panellists involved in hospital blood/sample transportation and communications, as these colleagues are critically important to the success of the MHP (e.g., ensuring a constant supply of blood packs from the bank). Given the numerous items lacking clinical trial evidence, our recommendations will need to be revisited and updated at regular intervals to evolve with this rapidly changing field of medicine. We note a particular lack of high-quality evidence for pediatric patients owing to exclusion of these patients from many clinical trials. 


\section{Conclusion}

We are confident that the 42 statements and 8 quality indicators, constructed through a highly structured process and with the involvement of a diverse and knowledgeable group of experts, will serve as a strong foundation for the creation of a robust MHP toolkit. The MHP toolkit, currently under development, will consist of recommendations and educational resources for 12 sections (patient transport; principals of damage control resuscitation; team dynamics; communication; laboratory testing; temperature management; use of blood components and adjuncts, including anticoagulant reversal; simulation and training tools; pediatric-specific recommendations; patient communication follow-up tools; research goals; and quality metrics and reporting) written by an expert working group, to be published and freely accessible in the spring of 2020 on the Ontario Regional Blood Coordinating Network website (http://transfusionontario.org/en/) and presented at the network's spring Transfusion Committee Forum. We expect that, with the use of the toolkit, hospitals will achieve higher adoption of evidence-based care of the patient with massive hemorrhage, improved speed of delivery of blood components and hemostatic adjuncts, and more diligent monitoring of clinical and laboratory parameters. There is an opportunity to track patient outcomes in existing prospectively collected databases in trauma and obstetrics and for inpatients held by the Canadian Institutes for Health Information in Ontario to understand the impact of this effort to standardize the care of these complex, high-acuity patients.

\section{References}

1. Harvin JA, Maxim T, Inaba K, et al. Mortality after emergent trauma laparotomy: a multicenter, retrospective study. 7 Trauma Acute Care Surg 2017;83:464-8.

2. Creanga AA, Syverson C, Seed K, et al. Pregnancy-related mortality in the United States, 2011-2013. Obstet Gynecol 2017;130:366-73.

3. Farooq N, Galiatsatos P, Aulakh JK, et al. Massive transfusion practice in nontrauma related hemorrhagic shock. 7 Crit Care 2018;43:65-9.

4. Spinella PC. Zero preventable deaths after traumatic injury: an achievable goal. 7 Trauma Acute Care Surg 2017;82:S2-8.

5. Tien HC, Spencer F, Tremblay LN, et al. Preventable deaths from hemorrhage at a level I Canadian trauma center. 7 Trauma 2007;62:142-6.

6. Deneux-Tharaux C, Saucedo M. Epidemiology of maternal mortality in France, 2010-2012 [article in French]. Gynecol Obstet Fertil Senol 2017;45:S8-21.

7. Berg CJ, Harper MA, Atkinson SM, et al. Preventability of pregnancy-related deaths: results of a state-wide review. Obstet Gynecol 2005;106:1228-34.

8. Young PP, Cotton BA, Goodnough LT. Massive transfusion protocols for patients with substantial hemorrhage. Transfus Med Rev 2011;25:293-303.

9. Milligan C, Higginson I, Smith JE. Emergency department staff knowledge of massive transfusion for trauma: the need for an evidence-based protocol. Emerg Med 7 2011;28:870-2.

10. Khan S, Allard S, Weaver A, et al. A major haemorrhage protocol improves the delivery of blood component therapy and reduces waste in trauma massive transfusion. Injury 2013;44:587-92.

11. Nunez TC, Young PP, Holcomb JB, et al. Creation, implementation, and maturation of a massive transfusion protocol for the exsanguinating trauma patient. 7 Trauma 2010;68:1498-505.

12. Cotton BA, Dossett LA, Au BK, et al. Room for (performance) improvement: provider-related factors associated with poor outcomes in massive transfusion. f Trauma 2009;67:1004-12.

13. Treml AB, Gorlin JB, Dutton RP, et al. Massive transfusion protocols: a survey of academic medical centers in the United States. Anesth Analg 2017;124:277-81.

14. Chin V, Cope S, Yeh CH, et al. Massive hemorrhage protocol survey: marked variability and absent in one-third of hospitals in Ontario, Canada. Injury 2019;50:46-53.

15. Etchill E, Sperry J, Zuckerbraun B, et al. The confusion continues: results from an American Association for the Surgery of Trauma survey on massive transfusion practices among United States trauma centers. Transfusion 2016;56:2478-86.

16. Bawazeer $M$, Ahmed N, Izadi $H$, et al. Compliance with a massive transfusion protocol (MTP) impacts patient outcome. Injury 2015;46:21-8.
17. Cannon JW, Khan MA, Raja AS, et al. Damage control resuscitation in patients with severe traumatic hemorrhage: a practice management guideline from the Eastern Association for the Surgery of Trauma. 7 Trauma Acute Care Surg 2017;82:605-17.

18. Boulkedid R, Abdoul H, Loustau M, et al. Using and reporting the Delphi method for selecting healthcare quality indicators: a systematic review. PLoS One 2011;6:e20476.

19. Jünger S, Payne SA, Brine J, et al. Guidance on Conducting and REporting DElphi Studies (CREDES) in palliative care: recommendations based on a methodological systematic review. Palliat Med 2017;31:684-706.

20. Meyer E, Delaney M, Lin Y, et al. A reporting guideline for clinical platelet transfusion studies from the BEST Collaborative. Transfusion 2013;53:1328-34.

21. Humphrey-Murto S, Varpio L, Gonsalves C, et al. Using consensus group methods such as Delphi and Nominal Group in medical education research. Med Teach 2017;39:14-9.

22. Benz D, Balogh ZJ. Damage control surgery: current state and future directions. Curr Opin Crit Care 2017;23:491-7.

23. Germanos S, Gourgiotis S, Villias C, et al. Damage control surgery in the abdomen: an approach for the management of severe injured patients. Int $\mathcal{F}$ Surg 2008;6:246-52.

24. Rossaint R, Bouillon B, Cerny V, et al. The European guideline on management of major bleeding and coagulopathy following trauma: fourth edition. Crit Care 2016;20:100.

25. Khan S, Davenport R, Raza I, et al. Damage control resuscitation using blood component therapy in standard doses has a limited effect on coagulopathy during trauma hemorrhage. Intensive Care Med 2015;41:239-47.

26. Etchill EW, Myers SP, McDaniel LM, et al. Should all massively transfused patients be treated equally? An analysis of massive transfusion ratios in the nontrauma setting. Crit Care Med 2017;45:1311-6.

27. Teixeira PG, Inaba K, Karamanos E, et al. The survival impact of plasma to red blood cell ratio in massively transfused non-trauma patients. Eur $\mathcal{7}$ Trauma Emerg Surg 2017;43:393-8.

28. Gorlin JB, Peters J, Van Buren N, et al. The confusion continues: evolving nature of massive transfusion protocol practice may reflect lack of evidence to support a single solution that fits all. Transfusion 2017;57:1322-4.

29. Plackett TP, Cherry DC, Delk G, et al. Clinical practice guideline adherence during Operation Inherent Resolve. 7 Trauma Acute Care Surg 2017;83:S66-70.

30. Gillissen A, van den Akker T, Caram-Deelder C, et al. Coagulation parameters during the course of severe postpartum hemorrhage: a nationwide retrospective cohort study. Blood Adv 2018;2:2433-42.

31. Revert $M$, Rozenberg $P$, Cottenet J, et al. Intrauterine balloon tamponade for severe postpartum hemorrhage. Obstet Gynecol 2018;131:143-9.

32. Jairath V, Kahan BC, Logan RF, et al. Outcomes following acute nonvariceal upper gastrointestinal bleeding in relation to time to endoscopy: results from a nationwide study. Endoscopy 2012;44:723-30.

33. Shemesh E, Czerniak A, Klein E, et al. A comparison between emergency and delayed endoscopic injection sclerotherapy of bleeding esophageal varices in nonalcoholic portal hypertension. 7 Clin Gastroenterol 1990;12:5-9.

34. Karkouti K, Callum J, Wijeysundera DN, et al. Point-of-care hemostatic testing in cardiac surgery: a stepped-wedge clustered randomized controlled trial. Circulation 2016;134:1152-62.

35. Lee AC, Reduque LL, Luban NL, et al. Transfusion-associated hyperkalemic cardiac arrest in pediatric patients receiving massive transfusion. Transfusion 2014;54:244-54.

36. Girelli G, Antoncecchi S, Casadei AM, et al. Recommendations for transfusion therapy in neonatology. Blood Transfus 2015;13:484-97.

37. Muszynski JA, Reeder RW, Hall MW, et al. RBC transfusion practice in pediatric extracorporeal membrane oxygenation support. Crit Care Med 2018;46:e552-9.

38. Howarth C, Banerjee J, Aladangady N. Red blood cell transfusion in preterm infants: current evidence and controversies. Neonatology 2018;114:7-16.

39. Hilton G, Daniels K, Goldhaber-Fiebert SN, et al. Checklists and multidisciplinary team performance during simulated obstetric hemorrhage. Int 7 Obstet Anesth 2016;25:9-16.

40. Delaney M, Roberts J, Mazor R, et al. Bleeding emergencies in neonatal and paediatric patients: improving the quality of care using simulation. Transfus Med 2018;28:405-12.

41. Kamerer JL. Massive transfusion protocol simulation: compound femur fracture and hypovolemic shock. Simul Healthc 2012;7:196-200.

42. Brydges R, Hatala R, Zendejas B, et al. Linking simulation-based educational assessments and patient-related outcomes: a systematic review and metaanalysis. Acad Med 2015;90:246-56.

43. Gjeraa K, Moller TP, Ostergaard D. Efficacy of simulation-based trauma team training of non-technical skills. A systematic review. Acta Anaesthesiol Scand 2014;58:775-87.

44. Murphy M, Curtis K, Lam MK, et al. Simulation-based multidisciplinary team training decreases time to critical operations for trauma patients. Injury 2018; 49:953-8.

45. Josey K, Smith ML, Kayani AS, et al. Hospitals with more-active participation in conducting standardized in-situ mock codes have improved survival after inhospital cardiopulmonary arrest. Resuscitation 2018;133:47-52.

46. Maddry JK, Perez CA, Mora AG, et al. Impact of prehospital medical evacuation (MEDEVAC) transport time on combat mortality in patients with non- 
compressible torso injury and traumatic amputations: a retrospective study. Mil Med Res 2018;5:22.

47. Meyer DE, Vincent LE, Fox EE, et al. Every minute counts: time to delivery of initial massive transfusion cooler and its impact on mortality. 7 Trauma Acute Care Surg 2017;83:19-24.

48. Acker SN, Bredbeck B, Partrick DA, et al. Shock index, pediatric age-adjusted (SIPA) is more accurate than age-adjusted hypotension for trauma team activation. Surgery 2017;161:803-7.

49. Schroll R, Swift D, Tatum D, et al. Accuracy of Shock Index versus ABC score to predict need for massive transfusion in trauma patients. Injury 2018;49:15-9.

50. Meyer DE, Cotton BA, Fox EE, et al. A comparison of resuscitation intensity and critical administration threshold in predicting early mortality among bleeding patients: a multicenter validation in 680 major transfusion patients. $\mathcal{F}$ Trauma Acute Care Surg 2018;85:691-6.

51. Neff LP, Cannon JW, Morrison JJ, et al. Clearly defining pediatric massive transfusion: cutting through the fog and friction with combat data. 7 Trauma Acute Care Surg 2015;78:22-8, discussion 8-9.

52. Foster JC, Sappenfield JW, Smith RS, et al. Initiation and termination of massive transfusion protocols: current strategies and future prospects. Anesth Analg 2017;125:2045-55.

53. Briggs A, Raja AS, Joyce MF, et al. The role of nontechnical skills in simulated trauma resuscitation. 7 Surg Educ 2015;72:732-9.

54. McLaughlin CM, Wieck MM, Barin EN, et al. Impact of simulation-based training on perceived provider confidence in acute multidisciplinary pediatric trauma resuscitation. Pediatr Surg Int 2018;34:1353-62.

55. Gregg SC, Heffernan DS, Connolly MD, et al. Teaching leadership in trauma resuscitation: immediate feedback from a real-time, competency-based evaluation tool shows long-term improvement in resident performance. 7 Trauma Acute Care Surg 2016;81:729-34.

56. Khan S, Brohi K, Chana M, et al. Hemostatic resuscitation is neither hemostatic nor resuscitative in trauma hemorrhage. 7 Trauma Acute Care Surg 2014; 76:561-7, discussion 7-8

57. Johnsen BH, Westli HK, Espevik R, et al. High-performing trauma teams: frequency of behavioral markers of a shared mental model displayed by team leaders and quality of medical performance. Scand 7 Trauma Resusc Emerg Med 2017;25:109.

58. Graw JA, Eymann K, Kork F, et al. Risk perception of blood transfusions - a comparison of patients and allied healthcare professionals. BMC Health Serv Res 2018;18:122.

59. Schonewille H, Honohan A, van der Watering LM, et al. Incidence of alloantibody formation after $\mathrm{ABO}-\mathrm{D}$ or extended matched red blood cell transfusions: a randomized trial (MATCH study). Transfusion 2016;56:311-20.

60. Barty RL, Pai M, Liu Y, et al. Group O RBCs: Where is universal donor blood being used? Vox Sang 2017;112:336-42.

61. Zeller MP, Barty R, Dunbar NM, et al. An international investigation into AB plasma administration in hospitals: How many $\mathrm{AB}$ plasma units were infused? The HABSWIN study. Transfusion 2018;58:151-7.

62. Valentine J, Lane T, Miller N, et al. Two transfusion-related acute lung injury episodes in a recipient of $\mathrm{AB}$ plasma from multiparous donors. Ther Apher Dial 2013;17:640-2.

63. Brohi K, Singh J, Heron M, et al. Acute traumatic coagulopathy. 7 Trauma 2003;54:1127-30.

64. Chandler WL, Ferrell C, Trimble S, et al. Development of a rapid emergency hemorrhage panel. Transfusion 2010;50:2547-52.

65. Baxter J, Cranfield KR, Clark G, et al. Do lactate levels in the emergency department predict outcome in adult trauma patients? A systematic review. $\mathcal{F}$ Trauma Acute Care Surg 2016;81:555-66.

66. Bouzat P, Ageron FX, Charbit J, et al. Modelling the association between fibrinogen concentration on admission and mortality in patients with massive transfusion after severe trauma: an analysis of a large regional database. Scand 7 Trauma Resusc Emerg Med 2018;26:55.

67. Alam A, Olarte R, Callum J, et al. Hypothermia indices among severely injured trauma patients undergoing urgent surgery: a single-centred retrospective quality review and analysis. Injury 2018;49:117-23.

68. Margarido C, Ferns J, Chin V, et al. Massive hemorrhage protocol activation in obstetrics: a 5-year quality performance review. Int 7 Obstet Anesth 2019;38: $37-45$.

69. Jurkovich GJ, Greiser WB, Luterman A, et al. Hypothermia in trauma victims: an ominous predictor of survival. 7 Trauma 1987;27:1019-24.

70. Lester ELW, Fox EE, Holcomb JB, et al. The impact of hypothermia on outcomes in massively transfused patients. 7 Trauma Acute Care Surg 2019;86: 458-63.

71. Perlman R, Callum J, Laflamme C, et al. A recommended early goal-directed management guideline for the prevention of hypothermia-related transfusion, morbidity, and mortality in severely injured trauma patients. Crit Care 2016;20: 107.

72. Rajagopalan S, Mascha E, Na J, et al. The effects of mild perioperative hypothermia on blood loss and transfusion requirement. Anesthesiology 2008;108: 71-7.

73. Lundgren $\mathrm{P}$, Henriksson $\mathrm{O}$, Naredi $\mathrm{P}$, et al. The effect of active warming in prehospital trauma care during road and air ambulance transportation - a clinical randomized trial. Scand 7 Trauma Resusc Emerg Med 2011;19:59.
74. Fadeyi EA, Emery W, Simmons JH, et al. Implementation of a new blood cooler insert and tracking technology with educational initiatives and its effect on reducing red blood cell wastage. Transfusion 2017;57:2477-82.

75. Metcalf RA, Baker SA, Goodnough LT, et al. Transportation cooler interventions reduce plasma and RBC product wastage. Am 7 Clin Pathol 2016;146: $18-24$.

76. Gonzalez-Porras JR, Graciani IF, Perez-Simon JA, et al. Prospective evaluation of a transfusion policy of D+ red blood cells into D- patients. Transfusion 2008;48:1318-24.

77. Yazer MH, Triulzi DJ. Detection of anti-D in D- recipients transfused with D+ red blood cells. Transfusion 2007;47:2197-201.

78. Bhella S, Gerard L, Lin Y, et al. Obstetric and trauma database review at a single institution finds the optimal maternal age restriction for the transfusion of O- blood to women involved in trauma to be 45 years. Transfusion 2012;52: 2488-9.

79. Cid J, Lozano M, Ziman A, et al. Low frequency of anti-D alloimmunization following $\mathrm{D}+$ platelet transfusion: the Anti-D Alloimmunization after D-incompatible Platelet Transfusions (ADAPT) study. Br 7 Haematol 2015. 168:598-603.

80. Holcomb JB, Tilley BC, Baraniuk S, et al. Transfusion of plasma, platelets, and red blood cells in a 1:1:1 vs a 1:1:2 ratio and mortality in patients with severe trauma: the PROPPR randomized clinical trial. FAMA 2015;313:471-82.

81. Nascimento B, Callum J, Tien H, et al. Effect of a fixed-ratio $(1: 1: 1)$ transfusion protocol versus laboratory-results-guided transfusion in patients with severe trauma: a randomized feasibility trial. CMA7 2013;185:E583-9.

82. Mesar T, Larentzakis A, Dzik W, et al. Association between ratio of fresh frozen plasma to red blood cells during massive transfusion and survival among patients without traumatic injury. 7AMA Surg 2017;152:574-80.

83. Dzik WH, Blajchman MA, Fergusson D, et al. Clinical review: Canadian National Advisory Committee on Blood and Blood Products - Massive Transfusion Consensus Conference 2011: report of the panel. Crit Care 2011; $15: 242$.

84. Simpson E, Lin Y, Stanworth S, et al. Recombinant factor VIIa for the prevention and treatment of bleeding in patients without haemophilia. Cochrane Database Syst Rev 2012;(3):CD005011.

85. Ranucci M, Isgro G, Soro G, et al. Efficacy and safety of recombinant activated factor VII in major surgical procedures: systematic review and meta-analysis of randomized clinical trials. [discussion]. Arch Surg 2008;143:296-304.

86. Karkouti K, Callum J, Rao V, et al. Protocol for a phase III, non-inferiority, randomised comparison of a new fibrinogen concentrate versus cryoprecipitate for treating acquired hypofibrinogenaemia in bleeding cardiac surgical patients: the FIBRES trial. BMF Open 2018;8:e020741.

87. Galas FR, de Almeida JP, Fukushima JT, et al. Hemostatic effects of fibrinogen concentrate compared with cryoprecipitate in children after cardiac surgery: a randomized pilot trial. 7 Thorac Cardiovasc Surg 2014;148:1647-55.

88. Schöchl H, Nienaber U, Maegele $M$, et al. Transfusion in trauma: thromboelastometry-guided coagulation factor concentrate-based therapy versus standard fresh frozen plasma-based therapy. Crit Care 2011;15:R83.

89. Schlimp CJ, Voelckel W, Inaba K, et al. Impact of fibrinogen concentrate alone or with prothrombin complex concentrate (+/- fresh frozen plasma) on plasma fibrinogen level and fibrin-based clot strength (FIBTEM) in major trauma: a retrospective study. Scand 7 Trauma Resusc Emerg Med 2013;21:74.

90. Noga T, Bruce AA, Blain H, et al. Four-factor prothrombin complex concentrates in paediatric patients - a retrospective case series. Vox Sang 2016;110: 253-7.

91. Maskens C, Downie H, Wendt A, et al. Hospital-based transfusion error tracking from 2005 to 2010: identifying the key errors threatening patient transfusion safety. Transfusion 2014;54:66-73, quiz 65.

92. Strauss R, Downie H, Wilson A, et al. Sample collection and sample handling errors submitted to the transfusion error surveillance system, 2006 to 2015. Transfusion 2018;58:1697-707.

93. CRASH-2 Trial Collaborators; Shakur H, Roberts I, Bautista R, et al. Effects of tranexamic acid on death, vascular occlusive events, and blood transfusion in trauma patients with significant haemorrhage (CRASH-2): a randomised, placebo-controlled trial. Lancet 2010;376:23-32.

94. Woman Trial Collaborators. Effect of early tranexamic acid administration on mortality, hysterectomy, and other morbidities in women with post-partum haemorrhage (WOMAN): an international, randomised, double-blind, placebo-controlled trial. Lancet 2017;389:2105-16.

95. Gayet-Ageron A, Prieto-Merino D, Ker K, et al. Effect of treatment delay on the effectiveness and safety of antifibrinolytics in acute severe haemorrhage: a meta-analysis of individual patient-level data from 40138 bleeding patients. Lancet 2018;391:125-32.

96. Roberts I, Shakur H, Coats T, et al. The CRASH-2 trial: a randomised controlled trial and economic evaluation of the effects of tranexamic acid on death, vascular occlusive events and transfusion requirement in bleeding trauma patients. Health Technol Assess 2013;17:1-79.

97. Morrison JJ, Dubose JJ, Rasmussen TE, et al. Military Application of Tranexamic Acid in Trauma Emergency Resuscitation (MATTERs) Study. Arch Surg 2012;147:113-9.

98. Beno S, Ackery AD, Callum J, et al. Tranexamic acid in pediatric trauma: Why not? Crit Care 2014;18:313. 
99. Nishijima DK, Monuteaux MC, Faraoni D, et al. Tranexamic acid use in United States children's hospitals. 7 Emerg Med 2016;50:868-74 e1.

100. Roberts I, Coats T, Edwards P, et al. HALT-IT - tranexamic acid for the treatment of gastrointestinal bleeding: study protocol for a randomised controlled trial. Trials 2014;15:450.

101. Chang R, Holcomb JB. Implementation of massive transfusion protocols in the United States: the relationship between evidence and practice. Anestb Analg 2017;124:9-11.

102. Humphrey-Murto S, Varpio L, Wood TJ, et al. The use of the Delphi and other consensus group methods in medical education research: a review. Acad Med 2017;92:1491-8.

Affiliations: Departments of Laboratory Medicine and Molecular Diagnostics (Callum, Chin, Viveiros), Surgery (Nathens, Nascimento), Emergency Services (McDonald), Critical Care Medicine (Adhikari) and Anesthesia (Margarido), Sunnybrook Health Sciences Centre; Departments of Laboratory Medicine and Pathobiology (Callum, Pendergrast, Skeate, Pavenski), Anesthesia (McVey, Karkouti, Alam, Margarido), Surgery (Nathens, Nascimento, Rizoli) and Paediatrics (Beno), University of Toronto; Division of Emergency Medicine (Yeh, Petrosoniak, McDonald, MacDonald), Department of Medicine, University of Toronto; Departments of Emergency Medicine (Petrosoniak), Surgery (Rizoli) and Laboratory Medicine (Sholzberg, Pavenski), St. Michael's Hospital; Department of Anesthesia and Pain Medicine (McVey, Skelton), The Hospital for Sick Children; Ontario Regional Blood Coordinating Network (Cope, Thompson, Collins, Owens); Department of Anesthesia and Pain Management (Karkouti), Sinai Health System, University Health Network, and Women's College Hospital, Toronto, Ont.; Department of Anesthesiology and Pain Medicine (Murto), Children's Hospital of Eastern Ontario; Department of Anesthesiology and Pain Medicine (Murto), University of Ottawa, Ottawa, Ont.; Paediatric Emergency Medicine (Beno), The Hospital for Sick Children; Department of Clinical Pathology (Pendergrast), University Health Network, Toronto, Ont.; Ornge Transport Medicine (McDonald, MacDonald), Mississauga, Ont.; Interdepartmental Division of Critical Care Medicine (Adhikari), University of Toronto; Department of Anesthesia (Alam, Arnold), North
York General Hospital, Toronto, Ont.; McMaster Centre for Transfusion Research (Arnold, Pai, Zeller); Departments of Medicine (Pai, Zeller) and Pathology and Molecular Medicine (Pai), McMaster University, Hamilton, Ont.; Canadian Blood Services (Arnold, Skeate, White); St. Michael's Hospital (Barratt, Chaudhry, Harvey), Toronto, Ont.; Department of Surgery (Beckett), McGill University, Montréal, Que.; Canadian Forces Health Services (Beckett), Ottawa, Ont.; Sunnybrook Health Sciences Centre (Brenneman), Toronto, Ont.; General Surgery, Acute Care and Trauma (Lampron), The Ottawa Hospital; Departments of Surgery (Lampron), Medicine (Tinmouth) and Laboratory Medicine and Pathology (Tinmouth), Faculty of Medicine, University of Ottawa, Ottawa, Ont.; Trauma Program and Quality Assurance (McFarlan), St. Michael's Hospital, Toronto, Ont.; Departments of Pathology (Ruijs) and Surgery (Van Heest), William Osler Health Centre, Brampton, Ont.; Lakeridge Health Corporation (Syer), Oshawa, Ont.; Department of Critical Care (Theriault), Health Sciences North, Sudbury, Ont.; Division of Hematology (Tinmouth), The Ottawa Hospital; University of Ottawa Centre for Transfusion Research (Tinmouth), Ottawa Hospital Research Institute, Ottawa, Ont.; Canadian Blood Services (Zeller), Ancaster, Ont.

Contributors: All of the steering committee members contributed to the study design and data analysis and interpretation, drafted the manuscript and critically revised it for important intellectual content. All of the panel members contributed to data interpretation, drafted the manuscript and critically revised it for important intellectual content. All of the authors approved the final version to be published and agreed to act as guarantors of the work.

Funding: This work was supported by the Ontario Regional Blood Coordinating Network through the Ontario Ministry of Health and Long-Term Care and Canadian Blood Services.

Supplemental information: For reviewer comments and the original submission of this manuscript, please see www.cmajopen.ca/content/7/3/ E546/suppl/DC1. 